Revue Française de Civilisation Britannique

\title{
De l'interdisciplinarité et du comparatisme en civilisation britannique
}

Of Interdisciplinarity and Comparatism in British Civilisation

\section{Vincent Latour}

\section{CpenEdition}

Journals

Édition électronique

URL : http://journals.openedition.org/rfcb/2857

DOI : $10.4000 /$ rfcb. 2857

ISSN : 2429-4373

Éditeur

CRECIB - Centre de recherche et d'études en civilisation britannique

Référence électronique

Vincent Latour, « De l'interdisciplinarité et du comparatisme en civilisation britannique », Revue Française de Civilisation Britannique [En ligne], XXIV-1 | 2019, mis en ligne le 22 mars 2019, consulté le 01 mai 2019. URL : http://journals.openedition.org/rfcb/2857 ; DOI : 10.4000/rfcb.2857

Ce document a été généré automatiquement le 1 mai 2019.

\section{cc) (†)}

Revue française de civilisation britannique est mis à disposition selon les termes de la licence Creative Commons Attribution - Pas d'Utilisation Commerciale - Pas de Modification 4.0 International. 


\title{
De l'interdisciplinarité et du comparatisme en civilisation britannique
}

\author{
of Interdisciplinarity and Comparatism in British Civilisation
}

\author{
Vincent Latour
}

\section{Introduction}

1 Il est sans doute quelque peu paradoxal de se livrer à une réflexion sémantique, épistémologique et méthodologique sur ce qui, au regard des instances nationales de la recherche (CNRS, notamment) ne constitue pas à proprement parler une discipline scientifique.

2 La civilisation, dans l'acception qui nous préoccupe ici, est connue des représentants des sciences humaines (qu'ils reconnaissent, ou non, un caractère scientifique à la démarche du civilisationniste) mais très peu en dehors des cercles universitaires, même bien informés. Si cette méconnaissance peut être imputée au manque de reconnaissance institutionnelle dont souffre la discipline, il apparaît qu'elle peut également l'être à la difficulté même de définir la civilisation, terme dans l'emploi duquel les civilisationnistes (anglicistes, mais aussi germanistes ou hispanistes, notamment) sont relativement isolés, même s'il apparait dans l'intitulé de deux sections CNU en histoire (la $21^{\mathrm{e}}$ " Histoire, civilisation, archéologie et art des mondes anciens et médiévaux » et la $22^{\mathrm{e}}$ : « Histoire et civilisations : histoire des mondes modernes, histoire du monde contemporain, de l'art ; de la musique »). Le terme de « civilisation » n'est, de fait, pas des plus aisés. La difficulté à définir la civilisation n'est pas nouvelle. Elle a suscité des débats depuis ses origines. Ainsi, à la fin des années 1970, l'anthropologue Maurice Godelier, formé à l'école structuraliste de Claude Lévi-Strauss, fut sollicité par deux civilisationnistes (Serge Dunis, civilisation du Commonwealth et Jacques Grangé, civilisation britannique) pour se livrer à un exercice de réflexion sémantique et théorique sur la notion de civilisation, dans l'acception première du terme et dans l'autre, liée mais plus récente, renvoyant à la 
discipline qui venait d'élargir le champ de l'anglistique et était alors en cours de codification. Une historienne spécialiste des États-Unis, Marianne Debouzy, participait également à l'entretien, au début duquel les quatre protagonistes se posèrent la question suivante : "Peut-on faire un enseignement de civilisation sans interroger les acceptions du mot civilisation ? ${ }^{1}$ » Dans le contexte français, l'opposition ancienne entre une conception large et universaliste de la civilisation et une vision relativement restreinte de la culture, se limitant, comme le soulignent Andrée Shepherd, Jean-Paul et Cécile Révauger à «[...] un ensemble de normes canoniques, comprenant la familiarité avec certains textes classiques, la maitrise d'un niveau de langue et une approche déférente de l'ensemble ${ }^{2}$ " ont sans doute constitué un obstacle, initialement. En France, le champ des études anglophones, initialement très littéraire, s'est considérablement élargi au cours des quatre dernières décennies (en s'ouvrant à la civilisation, la linguistique, et, plus récemment, au LANSAD), même si l'intitulé de la $11^{\mathrm{e}}$ section du CNU (« Langues et littératures anglaises et anglosaxonnes ») ne reflète pas vraiment cette diversité et rappelle l'hégémonie passée de la littérature ${ }^{3}$. Pendant longtemps, la civilisation fut considérée comme une discipline accessoire de l'anglistique. Selon Monica Charlot, avant de gagner ses lettres de légitimité et de noblesse en faisant son entrée à l'agrégation d'anglais dans les années 1970, les enseignements de civilisation étaient plus descriptifs qu'analytiques : "Les cours de licence tendaient à être [...] relativement pauvres du point de vue conceptuel et sollicitaient surtout la mémoire, utilisant dates et faits sans souci de contextualisation ou d'historiographie ", les départements d'anglais enseignant " plutôt les institutions que la vie politique, le droit plutôt que le fait ${ }^{4}$ ».

3 Au sein de l'anglistique européenne, la civilisation (britannique, notamment), tant du point de vue de sa dénomination que de son objet, constitue une spécificité française. Dans les autres pays européens, les études anglaises sont très majoritairement dominées par la littérature et la " langue ", comme en témoigne, livraison après livraison, The European English Messenger, la revue de la European Society for the Study of English, envoyée au membres de la SAES. Les ateliers de civilisation qui se tiennent dans le cadre des congrès de l'ESSE ou de sociétés savantes analogues sont le plus souvent très (voire, exclusivement) franco-français.

4 La méconnaissance de notre discipline, en France et à l'étranger, conjuguée à l'absence de réel texte fondateur et à la relative carence de textes théoriques et méthodologiques en matière de «civilisation " à proprement parler (en dépit, notamment, des écrits remarquables de Monica Charlot, mentionnés plus haut ou ceux de François Poirier 5 ) plaident donc pour la tentative de délimitation épistémologique et de réflexion méthodologique à laquelle le présent auteur va tenter de se livrer ici.

Dans un premier temps, nous examinerons conjointement deux des principales caractéristiques de la civilisation: l'interdisciplinarité et l'extériorité paradoxale du civilisationniste vis-à-vis de son objet d'étude. Dans un deuxième temps, nous montrerons en quoi ces caractéristiques sont compatibles avec le comparatisme, dont les différentes modalités seront envisagées et dont on montrera les applications possibles dans le champ de la civilisation britannique. 


\section{L'interdisciplinarité : caractéristique décisive inhérente à la civilisation?}

6 L'interdisciplinarité est souvent considérée comme le trait le plus saillant de la civilisation. Néanmoins, pour indispensable, louable et fructueuse qu'elle soit, peut-on avancer que l'interdisciplinarité distingue de manière si décisive que cela les civilisationnistes des historiens, sociologues, politistes ou encore, anthropologues ? En effet, ces représentants des sciences sociales sont depuis fort longtemps confrontés, parfois à leur corps défendant, à l'hybridation de leurs spécialités respectives, et ce, dans certains cas, depuis les origines des disciplines en question. L'histoire, la sociologie, l'anthropologie et la science politique ont connu (et connaissent encore) d'étonnantes interpénétrations disciplinaires, de surprenantes hybridations, témoignages d'un mouvement général des sciences sociales vers l'interdisciplinarité. L'exemple de l'histoire, est, à cet égard, éloquent. Avec la montée en puissance de l'anthropologie, de la sociologie et de la science politique tout au long du XXe siècle, l'histoire, a ainsi été soumise, selon Nicolas offenstadt, " aux courants montants » de ces disciplines, tantôt pour s'en démarquer, tantôt, au contraire, pour se positionner en leur sein. Le résultat de cette mutation, a constitué, d'après ce même historien, "un bouleversement de l'ordonnancement traditionnel » selon lequel, de manière schématique, l'histoire avait pour objet le passé ; la sociologie, le présent ; l'anthropologie, les sociétés extra-occidentales ; la science politique, le gouvernement des hommes ${ }^{6}$.

7 Le champ de l'histoire ne saurait, en effet, être qualifié de monolithique. Il englobe désormais l'économie, la vie artistique, intellectuelle et sociale, le tout avec une diversification considérable des supports étudiés par l'historien, bien au-delà des seules sources écrites ${ }^{7}$. Ce mouvement, rappelle Nicolas offenstadt, fut amorcé, progressivement, dès l'entre-deux guerres, grâce aux Annales d'histoire économique et sociale (revue fondée en 1929), dans le premier numéro desquelles Marc Bloch fustigeait " les schismes redoutables " cloisonnant les différentes branches des sciences sociales ${ }^{8}$. L'alliance de l'histoire avec l'économie et la sociologie permettait, selon Marc Bloch et Lucien Febvre, autre animateur de la revue, de s'affranchir d'un certain académisme, celui de l'histoire politique " officielle», en l'occurrence".

Largement amorcée avant le Seconde Guerre, donc, cette mutation s'est poursuivie. Les historiens s'intéressent désormais à l' " histoire totale ${ }^{10}$ ", que Michel Vovelle, un des pères de la Nouvelle histoire, qualifie d'histoire « de la cave au grenier ", illustrant ainsi l'intérêt accru des historiens pour l'histoire culturelle depuis les années $1980^{11}$. Les années 1980 et 1990 furent également marquées par une grande perméabilité de certains courants de l'histoire à la sociologie de Pierre Bourdieu, qui ambitionnait d'étudier les mécanismes sociaux dans leur ensemble. La socio-histoire a ainsi été particulièrement influencée par la sociologie bourdieusienne. Elle s'intéresse notamment à l'opinion publique, ou, encore, à la formation de la Nation et témoigne, selon le terme de Nicolas Offenstadt, d'une " hybridation de l'histoire et de la sociologie ${ }^{12}$ ».

9 À elle seule, donc, l'interdisciplinarité ne saurait être considérée comme la caractéristique la plus décisive de la civilisation. D'ailleurs, en l'absence d'outils théoriques ou méthodologiques qui lui soient propres, avoir recours à ceux des autres sciences sociales n'est pas tant un choix qu'une obligation. 


\section{L'extériorité paradoxale du civilisationniste vis à vis de son champ d'étude}

10 Une autre caractéristique semble au moins aussi décisive que l'interdisciplinarité : l'extériorité du civilisationniste vis-à-vis de son champ d'étude. Andrée Shepherd, Cécile et Jean-Paul Révauger définissent la civilisation (britannique, en l'occurrence) comme " l'exploration distancée du champ culturel britannique par les chercheurs d'une autre culture et d'une autre nation ${ }^{13} "$.

11 Cette notion de distanciation (qui n'est toutefois pas l'apanage des seuls civilisationnistes ${ }^{14)}$ est fondamentale et constitue, sans nul doute, un des atouts majeurs du civilisationniste. Elle lui confère un point de vue original sur des faits sociaux ou historiques se rapportant au Royaume-Uni, et, le cas échéant, lui permet d'explorer des problématiques négligées dans le pays qu'il étudie et/ou de proposer des analyses ou des interprétations qui y ont peu cours ${ }^{15}$. Cependant, si elle est bien réelle, la distanciation du civilisationniste est paradoxale. En effet, c'est précisément la conjugaison de son extériorité " géographique » à son champ d'étude ${ }^{16}$ et de « l'intériorité » que lui confère sa connaissance fine de la langue et de la culture du pays étudié qui le distingue résolument des spécialistes des disciplines " classiques " des sciences humaines et sociales (sauf si, pour des raisons personnelles, ces derniers ont baigné durablement dans les deux cultures).

La distanciation paradoxale du civilisationniste vis-à-vis de son champ d'étude, caractéristique la plus déterminante du civilisationniste, prédispose au comparatisme et facilite la transition vers ce dernier. La sociologue Cécile Vigour souligne d'ailleurs dans son ouvrage théorique et méthodologique sur les comparaisons en sciences sociales que la combinaison distance / proximité est indispensable à la démarche comparatiste et en constitue même un des atouts majeurs. Elle avance en effet que «la mise à distance $d u$ chercheur par rapport à son objet d'étude est [...] impérieuse ${ }^{17}$ ", avant de citer F. Schultheis : " C'est en vertu de tels principes de ruptures et de mise en suspens cognitif que la stratégie comparative devient génératrice de doutes et de questions fructueuses ${ }^{18}$.»

13 Comme cela va être montré dans la section suivante, le comparatisme semble donc être non seulement l'allié naturel de la civilisation, mais, aussi, d'une certaine manière, sa prolongation logique et directe.

\section{De l'usage du comparatisme et des comparaisons en civilisation britannique}

La méthode comparative peut enrichir significativement les travaux des civilisationnistes, du fait de la complexification considérable induite par l'analyse comparative. Cécile Vigour, dont les travaux s'appuient notamment sur ceux de Neil J. Smelser ${ }^{19}$, souligne ainsi :

En replaçant son objet de recherche dans une perspective temporelle plus longue ou en le confrontant à d'autres réalités géographiques et culturelles, le comparatiste étend son champ d'observation. [...] La comparaison doit ainsi être conçue comme une démarche, un état d'esprit destiné à déplacer le regard du chercheur. Comparer, c'est en effet non seulement accepter de se décentrer, mais 
également rendre plus exigeants la formulation d'hypothèses et le travail de théorisation ${ }^{20}$. chercheurs qui n'y ont pas recours. Pour autant, si l'historienne Élise Julien souligne le caractère « naturel » des comparaisons, pratiquées hors démarche scientifique depuis la nuit des temps, le comparatisme relève quant à lui d'une démarche moins évidente qu'il n'y paraît :

La tendance à la comparaison est une pratique intellectuelle à la fois archaïque et universelle, au principe de toute analyse : on comprend d'autant mieux un objet qu'on le confronte à d'autres objets analogues ou différents. Le comparatisme va cependant au-delà de ce constat : il consiste à placer la comparaison au cœur de l'analyse ${ }^{21}$.

Cécile Vigour, souligne, quant à elle, qu'il y a eu finalement «[...] peu de réflexion sur l'usage et l'élaboration de la comparaison [...], comme si la comparaison allait de soi et ne nécessitait l'élaboration ni d'outils, ni d'une démarche spécifique ${ }^{22}$ ». Le flou théorique relatif qui entoure cette méthode et la complexité de sa mise en œuvre (ces deux points étant naturellement corrélés) plaident donc pour le travail sémantique et méthodologique qui va suivre.

\section{Définitions et typologie}

L'approche comparative a donné lieu à d'innombrables définitions, parfois contradictoires. On parle souvent de " comparaisons », terme qui désigne le travail concret effectué par le comparatiste, lorsqu'il contraste deux pays et /ou deux époques, par exemple.

Le comparatisme, quant à lui, désignera ici davantage la démarche globale, la méthode, voire, «la science des comparaisons ». Dans ce dernier cas, il est aux comparaisons ce que l'historiographie est à l'histoire (même si le comparatisme ne constitue pas une discipline, mais plutôt une méthode, une " boîte à outils ${ }^{23}$ " transdisciplinaire, comme le souligne Cécile Vigour).

18 La définition la plus générale du terme " comparaison ", celle donnée par le dictionnaire Le Petit Robert, n'est pas la plus mauvaise, bien qu'elle ne soit pas spécifique aux sciences sociales. Simple et fonctionnelle, elle décrit l'essentiel du mécanisme qui sous-tend la démarche du comparatiste, quelle que soit la discipline dont il est issu : « Le fait d'envisager ensemble deux ou plusieurs objets pour en chercher les différences ou les ressemblances ${ }^{24}$. »

Parmi la multitude d'autres définitions possibles, on en retiendra deux. La première est celle des sociologues Jean-Renaud Lambert et Sandrine Parayre, selon qui la démarche comparative « vise à situer un objet de recherche dans des cadres divers pour en faire apparaître les caractéristiques invariantes et celles qui dépendent du contexte ${ }^{25} »$. Cette définition permet de rendre compte de la mise en perspective (historique et / ou géographique, notamment) inhérente à l'approche comparatiste.

Le choix de l'objet à comparer est naturellement déterminant: c'est là qu'intervient l'autre définition retenue, énoncée par Cécile Vigour :

Comparer, c'est [...] relever des différences et des points communs en fonction d'un critère qu'il convient de définir au préalable et qui oriente le regard du chercheur. Dès lors que l'on considère au moins deux termes en fonction d'un même critère, $a$ priori, rien n'est incomparable ${ }^{26}$. 
21 La notion de " critère " est bien entendu fondamentale, car de ce critère découlera la stratégie comparative adoptée par le chercheur. Or, aborder un objet de comparaison sans stratégie comparative réelle ou avec un angle comparatif biaisé est voué à l'échec. Il s'agit même d'un des écueils majeurs de la démarche comparative. Préalablement à toute comparaison, pour pouvoir la mener à bien, il faut donc s'assurer d'avoir identifié un critère suffisamment solide. Comme le souligne l'historienne Élise Julien, il convient de ne pas comparer des objets en apparence incomparables (« des pommes avec des poires », si j'ose dire) pour, in fine, arriver à la conclusion qu'effectivement, ils le sont bien. Dans ce cas-là, la comparaison tourne court et même, tourne à vide ${ }^{27}$. Il convient à présent de dresser une typologie des principales comparaisons possibles.

Les deux principaux types de comparaisons, dans le temps et dans l'espace, feront l'objet d'un traitement conjoint, pour des raisons qui seront exposées ci-après. Les comparaisons dans le temps sont incontestablement celles qui sont les plus fréquemment mobilisées dans le champ de la civilisation, y compris par des civilisationnistes ne se définissant pas comme comparatistes mais percevant l'intérêt d'une approche diachronique. L'intérêt scientifique de replacer les faits sociaux dans une perspective historique plus large en fait apparaître, pour reprendre certains des termes de la définition de Jean-Renaud Lambert et Sandrine Parayre déjà citée, " les caractéristiques invariantes " et " celles qui dépendent du contexte ». Dans les travaux de la plupart des comparatistes spécialistes de sciences sociales, les comparaisons dans le temps et dans l'espace ne constituent pas des catégories étanches. À cet égard, Cécile Vigour note qu'à côté de l'approche diachronique, chère à l'école des Annales, en existe une autre, dite " synchronique ", qui vise à étudier un phénomène à la même époque, à l'échelle d'un pays entier, de deux pays, voire, d'un continent (ex. le monachisme en Europe au Moyen Âge, par exemple ${ }^{28}$. La combinaison des approches diachronique et synchronique est possible et fréquente en histoire, mais également en civilisation britannique, à l'évidence. Les comparaisons entre groupes ou « communautés " (ex. intégration socio-économique; modalités de mobilisation; nature des liens avec le gouvernement) constituent également une forme de comparaison possible. Il est bien entendu possible et même très fréquent de coupler au moins deux types de comparaisons (dans le temps et dans l'espace, notamment) : on parle alors de comparaisons « multivariée », pour citer un terme employé par Cécile Vigour ${ }^{29}$.

\section{De l'usage du comparatisme en civilisation britannique : étude de cas et application possible}

23 En se fondant sur les deux définitions du comparatisme retenues plus haut, nous tenterons de schématiser la démarche du civilisationniste comparatiste, à l'aide d'un diagramme composé d'une série de cercles concentriques qui me semble à même de rendre compte des « cadres » mentionnés dans la définition de Jean-Renaud Lambert et Sandrine Parayre.

Cette illustration méthodologique prendra appui sur un cas concret: la lutte contre la discrimination raciale au Royaume-Uni. Le critère de comparaison, qui est donc, le centre commun aux quatre cercles, est la prise en compte (ou non) de la question de la discrimination raciale par les gouvernants, aux niveaux national et supranational.

Le Royaume-Uni a adopté, dès le milieu des années 1960, une attitude très volontariste en matière de lutte contre la discrimination raciale, couplée à des mesures de restriction de 
l'immigration. Ce volontarisme était en partie inspiré par le contexte américain, pourtant très différent. Malgré des tensions raciales qu'il convient de ne pas minimiser, la GrandeBretagne wilsonienne n'était pas les États-Unis des Civil Rights; les immigrés du nouveau Commonwealth de Bradford ou Smethwick n'étaient pas les Noirs du Mississippi, ou du Bronx. Pour autant, la peur de voir le pays s'embraser de la même manière que certains quartiers américains (émeutes de Watts, 1965) était réelle. Il faut donc garder l'influence du contexte américain à l'esprit lorsque l'on évoque la mise en œuvre de la législation ad hoc dite sur les "relations raciales ", même s'il est indispensable d'être conscient des limites d'une telle comparaison.

Le diagramme indique également qu'avec ses politiques publiques, le Royaume-Uni s'est inscrit dans une dynamique supranationale de lutte contre la discrimination, qu'il a légèrement anticipée, (voire, influencée, même si cela reste à établir). Par contraste, la France de 1965, dont l'approche de l'immigration était purement économique, demeurait pour l'essentiel indifférente à la question de l'intégration, qui tout au plus, commençait à se poser sous l'angle humanitaire et sanitaire (loi Debré sur les bidonvilles, 1964) : il est donc logique que les premières mesures de lutte contre le racisme et la discrimination raciale aient été prises dans l'hexagone sept années plus tard qu'outre-Manche.

En ce qui concerne la schématisation via le diagramme, donc, un premier niveau de comparaison, matérialisé par le deuxième cercle, permet de mettre en évidence la quasisimultanéité de l'adoption du Race Relations Act de 1965 avec l'instauration de l'affirmative action aux États-Unis, dans le contexte du mouvement des Civil Rights.

Le troisième cercle fait apparaître le niveau supranational, avec, en décembre 1965, l'adoption par l'assemblée générale des Nations Unies de la Convention internationale sur l'élimination de toutes les formes de discrimination raciale (CIETFDR ${ }^{30}$ ), qui allait entrer en vigueur en 1969.

Le quatrième dernier cercle enfin, rend compte de l'évolution, lente mais réelle, de la législation française en la matière, avec la « Loi 72-546 du $1^{\mathrm{er}}$ juillet 1972 relative à la lutte contre le racisme ", sanctionnant toute incitation à la discrimination, à la haine et à la violence pour des motifs ethniques, nationaux, raciaux et religieux, adoptée un an après la ratification par la France de la Convention internationale sur l'élimination de toutes les formes de discrimination raciale (que le Royaume-Uni avait ratifiée dès $1966^{31}$ ). Le schéma qui suit, bien que très rudimentaire dans sa conception, permet de constater que si l'on s'en tient strictement au niveau national (et donc, sur le diagramme, au plus petit des quatre cercles) l'essentiel nous échappe. La vision qui résulterait d'une analyse non comparative de la question de la lutte contre la discrimination raciale (comme de tant d'autres) ne serait donc qu'extrêmement partielle et n'aurait qu'un intérêt scientifique très limité, réduit au niveau descriptif le plus basique.

a démarche comparative, si elle est correctement menée, complexifie donc considérablement l'analyse, ce qui constitue un grand atout pour l'universitaire, tant dans ses écrits que dans ses enseignements. Error: Reference source not found

Le chercheur devra néanmoins prendre garde, comme le souligne Élise Julien, à ne pas « conforter les clivages nationaux, à pétrifier les oppositions, sans voir les interférences et les dynamiques qui peuvent se produire ${ }^{32} »$.

33 En effet, un des écueils possibles des comparaisons (et, peut-être, une des principales sources de défiance à leur égard) est de les utiliser comme des prétextes afin, dans le cas 
des comparaisons internationales, de figer les "modèles" et in fine, d'en légitimer certains au détriment d'autres.

\section{De la légitimité des civilisationnistes à investir le champ comparatiste} légitimement intimider ou décourager les civilisationnistes. En matière de comparaisons internationales, à l'écrit, à partir de critères de comparaison clairement identifiés, il s'agit d'entrecroiser des éléments ayant trait à plusieurs pays, en évitant l'écueil de la juxtaposition et donc, de la fausse comparaison, ou, du moins, de la comparaison incomplète, qui aboutirait à plusieurs blocs monolithiques, même si, dans un article comme dans un ouvrage ou un numéro de revue, ce désagrément peut être limité par la présence d'une introduction et d'une conclusion générales comparatives. Néanmoins, ce n'est pas toujours le cas et nombre d'ouvrages, surtout collectifs, revendiquent leur dimension comparative en proposant, en fait, une succession de contributions strictement nationales, laissant le soin au lecteur d'effectuer la comparaison lui-même, en tirant les conclusions de points présentés isolément. Enfin, d'un point de vue pratique, dans un colloque, il est souvent compliqué de présenter une communication comparative en vingt minutes : le faire implique, à n'en pas douter, une discipline plus importante que pour une communication classique.

\section{Conclusion}

Dans un premier temps, nous avons insisté sur l'atout considérable du civilisationniste par rapport aux représentants des sciences sociales classiques, à savoir la combinaison de son extériorité (certes relative et variable) vis-à-vis de son objet d'étude, et l'intériorité 
que lui confère sa connaissance du pays, de sa culture et de sa langue. Cette spécificité est sans nul doute plus décisive encore que l'interdisciplinarité (fréquemment mise en avant, à juste titre), qui, quoi qu'indispensable au civilisationniste, ne lui est pas propre, toutes les sciences sociales étant concernées, peu ou prou, par un mouvement vers l'interdisciplinarité, voire, vers l'hybridation disciplinaire. L'interdisciplinarité et la combinaison intériorité / extériorité inhérentes au civilisationniste facilitent grandement le recours au comparatisme. Pour être mis correctement en œuvre, il suppose, préalablement, l'identification d'un critère objectif de comparabilité, sous peine de courir à l'échec, divers types de comparaisons pouvant être mobilisés conjointement. L'approche comparative peut reposer sur une comparaison point par point (dans ce cas, toutes les sections d'un article ou d'un ouvrage seront comparatifs) ou se fonder sur quelques points de comparaison, sans doute plus aisée à mettre en œuvre mais dont l'intérêt scientifique est néanmoins important.

Cela nous a conduits, en dernier lieu, à montrer les usages possibles du comparatisme en civilisation britannique et à mettre en évidence, pour certaines questions, les limites d'une approche non comparative.

Les réticences des anglicistes vis-à-vis du comparatisme tiennent sans doute aussi en grande partie à ce qu'ils perçoivent comme leur manque de compétence ou de légitimité scientifique pour travailler sur un autre pays que celui dont ils sont spécialistes, même si cet autre pays est le leur. Cette deuxième raison, cela va de soi, découle de la précédente : ils sont d'autant plus rétifs, qu'ils n'ont pas été formés au comparatisme.

Ce sentiment d'illégitimité voire, parfois, de complexe vis-à-vis des représentants des disciplines reconnues comme des sciences sociales à part entière, caractérise encore fréquemment l'attitude des civilisationnistes en général, même lorsqu'ils ne sont pas comparatistes. D'une certaine manière, l'autocensure est sans doute plus néfaste à la civilisation que l'hostilité, réelle ou supposée, des historiens, sociologues ou politistes à l'égard de notre discipline.

41 Il ne s'agit bien évidemment pas de nier les difficultés liées à la conversion vers le comparatisme, tant du point de vue thématique que méthodologique. Néanmoins, le recours au comparatisme permet au chercheur français de collaborer plus facilement avec ses homologues britanniques (d'autant plus si ces derniers sont eux-mêmes comparatistes), demandeurs de comparaisons (ou de regards extérieurs sur des faits sociaux ou historiques). Cela permet aux civilisationnistes de mieux valoriser leurs travaux et donc, de donner à ces derniers, ainsi qu'à la civilisation en général, un rayonnement bien plus important ${ }^{33}$. Incontestablement, en dernière analyse, les civilisationnistes jouissent d'une bien meilleure reconnaissance que la civilisation. Ils sont reconnus comme des spécialistes compétents de leur champ d'étude et c'est pour cela qu'ils sont sollicités pour des collaborations scientifiques, tant par des spécialistes d'autres disciplines que par les médias, lorsque leurs analyses et leur regard d'expert sont jugés pertinents pour éclairer actualité ou histoire britanniques, par exemple.

Vincent Latour est Professeur des Universités en civilisation britannique contemporaine à l'Université Toulouse Jean Jaurès et chargé d'enseignements à l'IEP de Toulouse. Membre de l'équipe CAS (EA 801), ses travaux, souvent comparatistes, portent sur l'immigration et l'intégration au Royaume-Uni et en France, avec un intérêt particulier pour les mobilisations collectives, la place de la religion dans l'espace public et la lutte contre les discriminations. 


\section{BIBLIOGRAPHIE}

CHARLOT Monica, «Livre blanc sur la recherche en études anglophones. Rapport sur le secteur $\mathrm{n}$ - 9 : Civilisation Britannique », 2001, p.1.URL http://saesfrance.org/wp-content/ uploads/2016/01/livreblanc.pdf (lien actif le 15 juin 2018).

FERRÉOL Gilles (dir.), Dictionnaire de sociologie, Paris, Armand Colin, 2009.

GODELIER Maurice, «Sur la notion de civilisation », Revue Françaises d'études américaines, n³, avril 1977, p.1. URL : http://transatlantica.revues.org/528\#text (lien actif le 29 juin 2018).

GUEREÑA Jean-Louis, « Civilisationnistes ou historiens ? ", Cahiers de civilisation espagnole contemporaine [en ligne], 1 (2007). URL : http://ccec.revues.org/117 (lien actif le 16 juin 2018).

JULIEN Élise, « Le comparatisme en histoire. Rappels historiographiques et approches méthodologiques », Hypothèses, 2004/1, p. 1. URL : https://www.cairn.info/revuehypotheses-2005-1-page-191.htm (lien actif le 15 juin 2018).

Nations Unies, «Collection des traités » https://treaties.un.org/pages/overview.aspx? path=overview/overview/page1_fr.xml (lien actif le 14 juin 2018).

OFFENSTADT Nicolas, L'historiographie, Paris, PUF-Que Sais-Je, 2011.

POIRIER François, « Toute production humaine est analysable: objets, documents et représentations», Méthodologie universitaire EC1: Initiation aux méthodes en Sciences Humaines (1999). URL : http://www.univ-paris13.fr/ANGLICISTES/POIRIER/UE03D-01/POIRIERUE03D-01.htm (lien actif le 19 août 2012).

RÉVAUGER Cécile, RÉVAUGER Jean-Paul, SHEPHERD Andrée, Le mémoire de civilisation britannique en maitrise et en DEA, Bordeaux, Pessac, Presses Universitaires de Bordeaux, Collection « Parcours universitaire », 2000.

REY Alain et REY-DEBOVE Josette (dir.), Le Petit Robert. Dictionnaire alphabétique et analogique de la langue française, Paris, Le Robert, 2011.

SMELSER Neil J., Comparative methods in the social sciences, Englewood Cliffs, NJ, Prentice-Hall, 1976.

SOLOMOS John, Race and Racism in Britain, London, Macmillan, 1993.

VAN DAMME Stéphane, "Comprendre les Cultural Studies : une approche d'histoire des savoirs », Revue d'histoire moderne et contemporaine, 2004/5 no51-4bis, p. 53-54. URL : http://www.cairn.info/ revue-d-histoire-moderne-et-contemporaine-2004-5-page-48.htm (lien actif le 5 juin 2018).

VIGOUR Cécile, La comparaison dans les sciences sociales: Pratiques et méthodes, Paris, La DécouverteGuides Repères, 2005.

\section{NOTES}

1. GODELIER Maurice, "Sur la notion de civilisation », Revue Françaises d'études américaines, $\mathrm{n}^{\circ} 3$, avril 1977, p.1. URL : http://transatlantica.revues.org/528\#text (lien actif le 29 juin 2018). 
2. RÉVAUGER Cécile, RÉVAUGER Jean-Paul, SHEPHERD Andrée, Le mémoire de civilisation britannique en maîtrise et en DEA, Bordeaux, Pessac, Presses Universitaires de Bordeaux, Collection «Parcours universitaire », 2000, p.32.

3. Pas plus d'ailleurs que les intitulés de $12^{\mathrm{e}}$ («Langues et littératures germaniques et scandinaves »); $13^{\mathrm{e}}$ («Langues et littératures slaves ») $14^{\mathrm{e}}$ (« Langues et littératures romanes : espagnol, italien, portugais, autres langues romanes ») et $15^{\mathrm{e}}$ sections (" Langues et littératures arabes, chinoises, japonaises, hébraïques, d'autres domaines linguistiques »).

4. CHARLOT Monica, «Livre blanc sur la recherche en études anglophones. Rapport sur le secteur $\mathrm{n}^{\circ}$ 9: Civilisation Britannique », p.1, 2001.URL http://saesfrance.org/wp-content/ uploads/2016/01/livreblanc.pdf (lien actif le 15 juin 2018).

5. En attestent notamment ses enseignements méthodologiques, dont certains étaient encore accessibles en ligne il y a quelques années sur le site de l'Université Paris 13. Voir par exemple François Poirier, " Toute production humaine est analysable: objets, documents et représentations", Méthodologie universitaire EC1: Initiation aux méthodes en Sciences Humaines (1999). URL : http://www.univ-paris13.fr/ANGLICISTES/POIRIER/UE03D-01/POIRIERUE03D-01.htm (lien actif le 19 août 2012).

6. OFFENSTADT Nicolas, L'historiographie, Paris, PUF-Que Sais-Je, 2011, p. 62.

7. OFFENSTADT Nicolas, op.cit., L'historiographie, p. 27-28.

8. OFFENSTADT Nicolas, op.cit., L'historiographie, p. 66-67.

9. OFFENSTADT Nicolas, op.cit., L'historiographie, p. 67.

10. GUEREÑA Jean-Louis, op.cit., « Civilisationnistes ou historiens ?», p. 6.

11. OFFENSTADT Nicolas, op.cit., L'historiographie, p. 6.

12. OFFENSTADT, op.cit., L'historiographie, pp. 78-79.

13. RÉVAUGER Cécile, RÉVAUGER Jean-Paul, SHEPHERD Andrée, op.cit., Le mémoire de civilisation britannique en maîtrise et en DEA, p.7.

14. Cette analyse distancée s'applique, plus généralement, aux chercheurs étrangers un pays autre que le leur. Ainsi, la contribution d'historiens nord-américains à la compréhension de l'attitude de la France sous l'Occupation et des ressorts du régime de Vichy n'est plus à prouver, comme le montrent les travaux remarquables de l'Américain Robert Paxton, et, plus récemment, du Canadien Éric Jennings.

15. RÉVAUGER Cécile, RÉVAUGER Jean-Paul, SHEPHERD Andrée, op.cit., Le mémoire de civilisation britannique en maîtrise et en DEA, p.8.

16. Cette extériorité n'est toutefois pas absolue, compte tenu des séjours réguliers sur le terrain effectués par nombre de civilisationnistes, dont la présence outre-Manche demeure néanmoins sporadique et intermittente. Elle est bien évidemment encore moins absolue pour les collègues britanniques (ou d'origine britannique) spécialistes de civilisation britannique, même si après de nombreuses années passées en France, l'extériorité au champ qu'ils étudient devient aussi, dans une certaine mesure, une réalité.

17. VIGOUR Cécile, La comparaison dans les sciences sociales: Pratiques et méthodes, Paris, La Découverte-Guides Repères, 2005, p.103.

18. SCHULTHEIS F., « Comme par raison - comparaison n'est pas toujours raison. Pour une critique sociologique de l'usage social de la comparaison interculturelle ", In Droit et Société, $\mathrm{N}^{\circ}$ 11-12, 1989, p. 221-222, cité dans VIGOUR Cécile, op.cit., La comparaison dans les sciences sociales: Pratiques et méthodes, p. 102-103.

19. Neil J. Smelser (1930-2017) qui s'est lui-même appuyé sur les travaux pionniers de Tocqueville, Durkheim et Weber, s'est intéressé aux comparaisons et aux modalités de leur mise en œuvre en sociologie, science politique, histoire ou économie. Voir SMELSER Neil J., Comparative methods in the social sciences, Englewood Cliffs, NJ, Prentice-Hall, 1976.

20. VIGOUR Cécile, op.cit., La comparaison dans les sciences sociales : Pratiques et méthodes, p.17-18. 
21. JULIEN Élise, "Le comparatisme en histoire. Rappels historiographiques et approches méthodologiques ",

Hypothèses, 2004/1, p. 1. URL : https://www.cairn.info/revue-hypotheses-2005-1-page-191.htm (lien actif le 15 juin 2018).

22. VIGOUR Cécile, op.cit., La comparaison dans les sciences sociales : Pratiques et méthodes, p. 18.

23. Ibid.

24. Le Petit Robert. Dictionnaire alphabétique et analogique de la langue française, REY Alain et REYDEBOVE Josette (dir.), Paris, Le Robert, 2011, p. 484.

25. LAMBERT Jean-Renaud et PARAYRE Sandrine, Lexique de sociologie, Paris, Dalloz, 2010, p. 49.

26. VIGOUR Cécile, op.cit., La comparaison dans les sciences sociales: Pratiques et méthodes, p. 7.

27. JULIEN Élise, op.cit., « Le comparatisme en histoire. Rappels historiographiques et approches méthodologiques ",

p. 198.

28. VIGOUR Cécile, op.cit., La comparaison dans les sciences sociales: Pratiques et méthodes, p. 53.

29. VIGOUR Cécile, op.cit., La comparaison dans les sciences sociales: Pratiques et méthodes, p. 13.

30. SOLOMOS John, Race and Racism in Britain, London, Macmillan, 1993, p. 79.

31. Site web des Nations Unies - «Collection des traités " https://treaties.un.org/pages/ overview.aspx?path=overview/overview/page1_fr.xml (lien actif le 14 juin 2018).

32. JULIEN Élise, op.cit., « Le comparatisme en histoire. Rappels historiographiques et approches méthodologiques ", p.198.

33. RÉVAUGER Cécile, RÉVAUGER Jean-Paul, SHEPHERD Andrée, op.cit., Le mémoire de civilisation britannique en maîtrise et en DEA, p.34.

\section{RÉSUMÉS}

Cet article propose une délimitation épistémologique et une réflexion méthodologique sur la civilisation en tant que discipline. Dans un premier temps, il examinera deux des principales caractéristiques de la civilisation: l'interdisciplinarité et l'extériorité paradoxale du civilisationniste vis-à-vis de son objet d'étude. Dans un dernier temps, il montrera en quoi ces caractéristiques sont compatibles avec le comparatisme, dont les différentes modalités seront envisagées et dont on montrera les applications possibles dans le champ de la civilisation britannique.

This article intends to delineate the contours, both epistemological and methodological, of civilisation as an academic discipline. First, two of the chief characteristics of civilisation shall be examined: interdisciplinarity and the "paradoxical externality" of civilisation specialists. Then, it will be shown that these characteristics are compatible with comparatism, whose various forms and possible applications to the field of civilisation will be considered.

INDEX

Mots-clés : civilisation britannique, interdisciplinarité, extériorité paradoxale, comparatisme Keywords : Civilisation, interdisciplinarity, paradoxical externality, comparative studies 


\section{AUTEUR}

\section{VINCENT LATOUR}

Cultures Anglo-Saxonnes, CAS (EA 801), Université Toulouse - Jean Jaurès, 31058 Toulouse Cedex 9. 